\title{
SAFENESS BY DESIGN: A NEW DESIGN PARADIGM?
}

\author{
lan de VERE, Ross McLEOD and Malte WAGENFELD \\ RMIT University
}

\begin{abstract}
Design has the potential to significantly improve safeness and wellbeing, and to identify and reduce risk either during the design phase or through targeted design interventions concerning product, infrastructure, systems, and services. The broad user-centred skills and technical knowledge base of designers allows for clear problem definition using ethnographic discovery processes, and creative design and innovative design resolution in a socio-technical context.

As designers' transition from product dependent (and market driven) outcomes to fewer tangible activities, increasingly the role of design as an enabler of societal wellbeing, capable of making a greater contribution to communities and lifestyles, opens up new practice foci. Whilst design has always been required to address safety from a compliance and/or product liability perspective, 'Safeness by Design' aims to apply an explicit safeness lens to design practice. Aspirational in its intent, it seeks to operate outside of safety compliance frameworks; utilising human centred design, experience and interaction design, social design, and service design approaches, rather than risk management methodologies, to achieve actual or perceived safeness.

This paper suggests Safeness by Design as a new design paradigm, examining the contribution of early works to safer urban contexts, and proposes a safeness-led approach to design practice.
\end{abstract}

\section{Keywords: Safeness, social innovation, design pedagogy, design intervention}

\section{INTRODUCTION}

Design has the potential to significantly improve safeness and wellbeing, and to identify and reduce risk either during the design phase or through targeted design interventions concerning product, infrastructure, systems, and services. The Lloyd's Register Foundation's Foresight Review of Design for Safety [1] asks whether design for safety can be used as a strategic tool, advocating the need for safety and risk reduction to be "considered at the earliest stages of developing new products, systems and technologies instead of reacting to failures that have taken place." Developing such a culture will require a holistic approach to understanding the myriad influences that impact safety including human behaviours, cultural and environmental contexts, and complex interactions.

Many industries have of course considered safety for a considerable time, prompted by incidents and failures, and the need for regulatory compliance and risk management, and these industries are heavily regulated from a safety perspective, for example the construction industry and other hazardous workplace environments. But whilst many of these initiatives have applied an occupational health and safety approach to accident and injury prevention, e.g., through mandating the use of PPE and compliance with safe practice and procedures, it is only more recently that mental health, worker performance and behavioural aspects have been included in these narratives. Similarly, the widely used Failure Mode and Effects Analysis (FMEA), whilst a valuable risk management conducted (along with other safety integration processes) in the later phase of design process to respect safety directives, does not replace the need for a comprehensive and strategic design approach to safeness. Houssin and Coulibaly [2] identified that safety integration typically occurs retrospectively by imposing hazard reduction procedures and constraints, rather than applying a user-centred and human behavioural understanding approach during the design process. As noted by the Lloyd's Foresight Review [1] "design for safety goes beyond legislation, regulations and standards." There is a critical need for a greater understanding of the potential for a safety or safeness led approach to design practice, the formulation or identification of appropriate design methods and the integration of this approach into design education. The Safeness by Design initiative aims to address this through targeted engagement with industry and government agencies to integrate a safeness agenda throughout the design curriculum. 


\section{SAFENESS BY DESIGN}

Safeness by Design, an initiative emerging from RMIT University's School of Design, aims to enhance health, wellbeing, and social values by using design to achieve actual and evident safeness across a broad range of environment and contexts. Rather than a design specialisation, we propose a new generalist and multidisciplinary approach to realise social impact through a safeness agenda. We advocate designers using their understanding of user behaviour and situational contexts to identify and respond to safeness concerns through targeted design interventions. It has been identified that whilst a strong focus on user-centred design has led to safer design solutions, user centred design is primarily focussed towards satisfying the user, instead of intrinsically creating safer designs [1]. To achieve a safer by design culture, it is apparent that we need to design for safer human behaviour and look beyond the mechanical and functional aspects of our products and service systems. We live in increasing complex worlds, where the risk of information and cognitive overload is a daily occurrence. Our multicultural societies require effective communication tools and a broader understanding of cultural behaviours and expectations. New technologies, whilst enabling, also pose new challenges from a safeness perspective. Designers need to understand the complexities of society, the inherent hazards within our environments and the risks to wellbeing posed by both cognisant and unconscious behaviours, regardless of intent.

\subsection{What is safeness?}

The pursuit of safeness can encompass many directions. Safeness can be defined as the state of not being exposed to danger or of protection from harm, freedom from hazards, danger, fear, risk, harm or loss, a feeling of safety and security. Whilst adverse impacts to safeness can result from either implicit or explicit risks, safeness is not purely in the physical; the perception of safeness is critical to wellbeing and confident societal behaviours. Glendon and Stanton [3] in their review of safety culture note that safety culture "is not merely empirical but rests upon a value decision." They found that improvement in safety was reflected in patterns of behaviour and working practices, as well as values, attitudes, and beliefs. Human behaviours and interactions can be unpredictable, and whilst safe practices can be embedded into workplaces, urban behaviours are more dynamic and diverse and require ethnographic research to inform the design process. Safeness by Design aims to employ design process and actions to remove vulnerability and insecurity and mitigate risk to achieve both perceived and actual safeness from a personal perspective across differing contexts, environments, and behaviours, through applying a holistic design approach. As noted by Sadeghi et al, it is important to assume a user behavioural model approach to safeness design, to "allow designing human activities in parallel with products, neither designing only products nor designing human activities after products [4].

\subsection{Establishing a new design paradigm - initial strategies}

For the initial stages of the Safeness by Design initiative, rather than take an occupational workplace or product category focus, the Safeness team elected to pursue a more holistic process of municipality engagement to identify and frame problems in existing urban landscapes and social contexts, and then propose a responsive design intervention. The reasons for this strategy were three-fold:

1. to identify and frame problems within a more local and accessible context,

2. to work quickly and broadly across multiple contexts with a social and community focus,

3. to evidence capability to enable early engagement in future urban planning or design cycles.

It was also felt that from an educational perspective, the opportunities for students to critically examine existing situations within their own urban environment and then propose an intervention, allowed for greater discourse on safeness (real and perceived) than would have been possible in a generative product design project. Whilst it is a long-term aim of this initiative to drive the introduction of safeness agendas into design cycles, it was felt that a responsive approach was more appropriate initially.

\section{DEVELOPING CAPABILITIES}

Addressing urban safeness in early studio collaborations with the City of Melbourne and the Ajuntament de Barcelona (city council), the initiative has resulted in design interventions that embrace holistic and multidisciplinary approaches to the issues of safeness on personal, social, spatial, and technological levels. Student project outcomes resulting from three collaborative research projects between designers at RMIT School of Design and the planners and strategists from two international cities, Barcelona and Melbourne have been exhibited in 2020/21 at both Melbourne and Barcelona Design Weeks and can be accessed through $\underline{\text { safenessbydesign.org. }}$. 


\subsection{Project One: Urban Safeness in Melbourne}

The pilot project for Safeness by Design emerged from a collaboration between the authors and urban planners and strategists from the City of Melbourne. Masters level design students in the 2019 Safe City Smart City project were tasked with identifying safeness concerns within the Melbourne metropolitan area, specifically the central business district and surrounding inner city suburbs.

The project challenged designers to uncover precedents and exemplars in safe urban design, conduct ethnographic research into perceptions of safety, and to evaluate the spatial and environmental conditions that promote safe behaviour. Students were given the opportunity to develop a stronger understanding of their city, to comprehend the specific behaviours of different user and mobility groups, and to identify areas where real and perceived safeness were lacking; and then to apply design intervention on personal, social, spatial, and technological levels. Design solutions aimed to ensure a 24-hour city that is safe, inclusive, and welcoming for all users, with a specific focus on personal mobility and the safety of women transiting the city at night.

Amongst the design proposals presented to city council planners and strategists were:

- $\quad$ an urban redevelopment that addressed conflicts between cyclist and pedestrian mobility in a heavily congested mixed-use promenade that is both a shopping and eating precinct and a commuter cyclist thoroughfare,

- an innovative app-based cycling route planning/navigation system that utilises data on traffic and cycling movement patterns to direct cyclists away from roads with heavier vehicular traffic and/or known hazards, towards safer cycling routes,

- a public safeness system that uses personal wearable technology to connect a user in danger with fellow citizens and/or police patrols, utilising a 'flash mob' approach for a safer community. This solution is an exemplar of new approaches to crime prevention and community activation,

- the 'How to be Considerate' educational campaign which explores the scenario of a stranger walking behind a woman at night and how this may increase fear; the outcome including a toolkit to encourage male awareness and behaviour change.
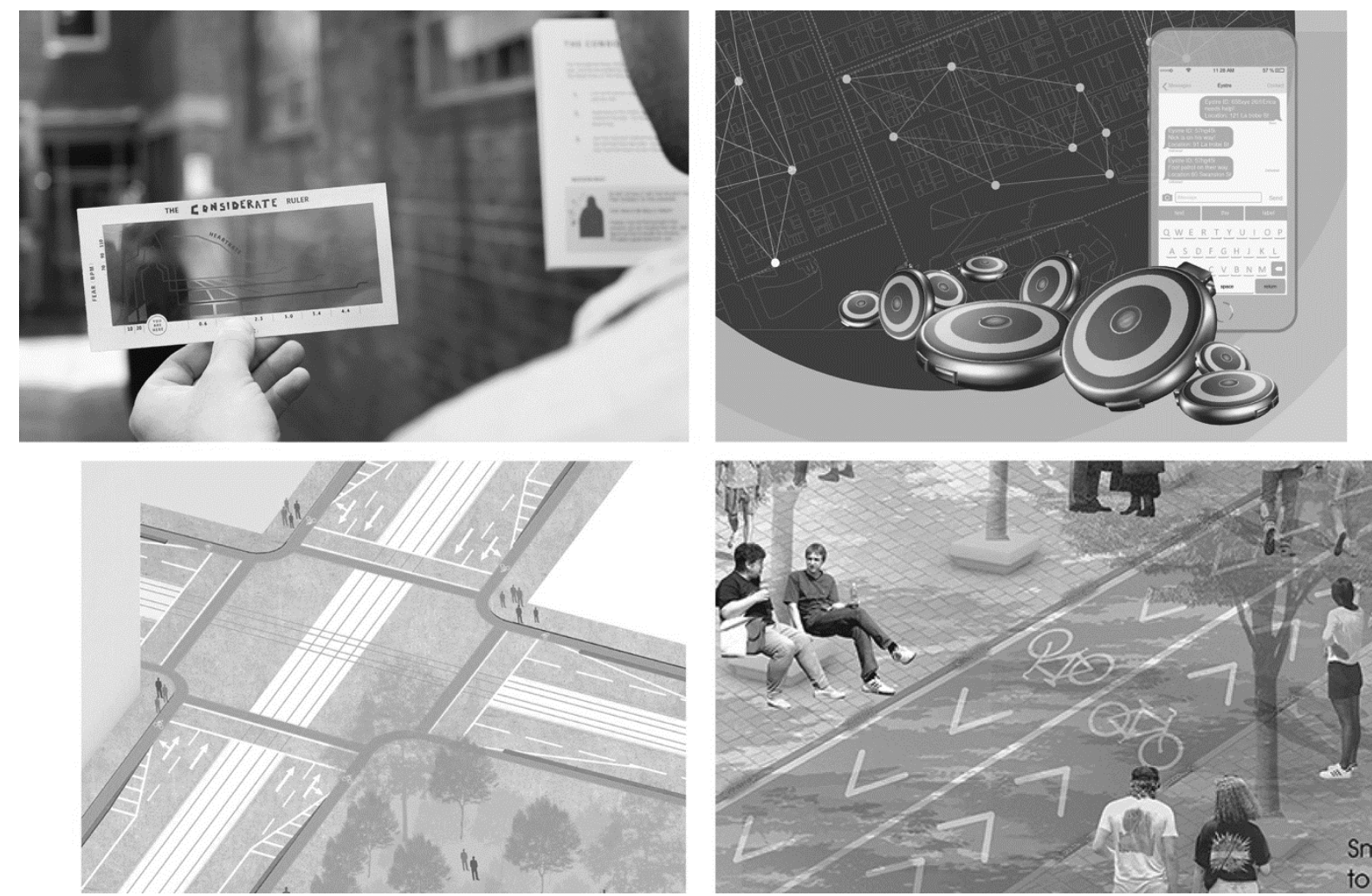

Figure 1. Safe City Smart City project design proposals (clockwise from top left: the 'How to be Considerate' campaign, Eystre wearable safeness technology, urban redesign to separate pedestrians and cyclists, new inner city cycle route planning and navigation 


\subsection{Project Two: e-scooters in Barcelona}

The introduction of electric kick scooters (e-scooters) posed a serious issue for the historic and busy metropolis of Barcelona. Since their introduction, e-scooters have caused significant concerns for city planners as they observed considerable speed disparity between riders and pedestrians, ignorance, and lack of compliance to local regulations, reckless and irresponsible rider behaviours, and urban clutter caused by the 'dock less' hire systems offered by unregulated service providers. These safeness concerns are exacerbated in Barcelona's crowded metropolis with its narrow streets and multitude of tourists.

In collaboration with planners and strategists from the Ajuntament de Barcelona (municipal council), Undergraduate and Masters design students addressed a range of safeness and logistical issues resulting from the rapid onset of these new personal micro mobility devices, including pedestrian and rider safety, helmet usage, e-scooter docking infrastructure, education, and rider behaviour change.

In doing so they attempted to empower the city to engage with e-scooter service providers with a clear set of operational guidelines and requirements to build a collaborative, yet regulatory relationship.

The Safe Mobility in Barcelona (2019-20) project delivered these innovative solutions to city planners:

- an educational and reward-based system that aims to create positive user behavioural change in escooter riders through rider training and usage (speed and behaviour) monitoring,

- an automated e-scooter smart navigation system that enhances pedestrian and e-scooter rider safety in congested areas, whilst empowering local communities and businesses to contribute to an e-scooter networked solution,

- $\quad$ an e-scooter infrastructure system including lockable scooter docking and charging systems that alleviates the core urban issues created by the dock less hire system,

- a safer e-scooter design with integrated helmet and safety compliance incorporating a cognitive test system that prevents intoxicated riding and speeding in pedestrian areas.
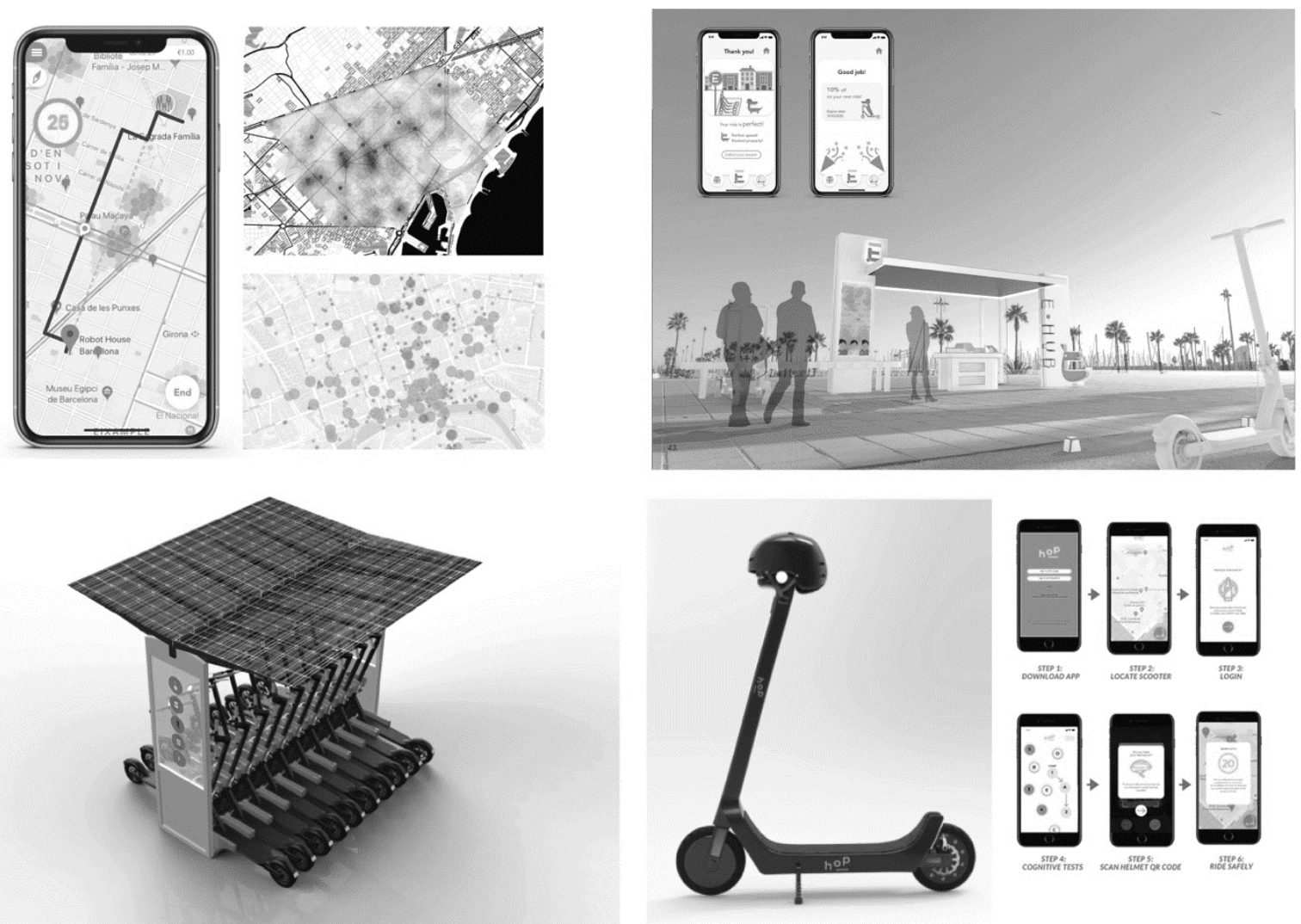

Figure 2. Safe Mobility in Barcelona project design proposals (clockwise from top left: automated smart navigation system, educational hub and reward-based behaviour change, safer e-scooter design with integrated helmet and safety compliance systems, scooter docking/charging hub 


\subsection{Project three: Safe Mobility with Covid-19}

The way we use and transit our cities will be significantly different for the foreseeable future. Coronavirus protective measures (especially social distancing and sanitation requirements) have resulted in unprecedented restrictions on personal mobility, generated unease and safeness concerns and a distrust of public transport and placed considerable pressure on cities and service providers. However, the impact of the global pandemic also provided an opportunity for designers to not only respond reactively to immediate community safeness needs, but to imagine safer, healthier post-pandemic cities; to consider how we can rethink our cities to shape new urban landscapes and enhance our social and professional interactions.

The 2020 Safe Mobility with Covid-19 project responded to emerging public safeness and mobility issues in environments defined by living with, and after, the Covid-19 pandemic. This project, which was conducted during the continuing pandemic lockdowns, realised solutions for coronavirus impacted urban landscapes with a focus on safe solutions for public transport, personal mobility, sanitation and contact avoidance, smart city technologies, and local communities.

In collaboration with the City of Melbourne and the Ajuntament de Barcelona, this project challenged Master's level design students to respond to the 'new normal' with design proposals that address the psychological, physical, and technical realities needed to ensure safe mobility in urban environments, whilst showcasing the value of human centred design and focused design interventions in responding to global challenges. Amongst the design interventions proposed to the city councils were:

- restoring trust in public transport through the design of digital and physical systems and services to maintain social distancing throughout transit journeys,

- using technological (smart city) solutions including real time crowd data mapping to provide route planning and navigation to maintain social distancing on our streets and reduce urban congestion hotspots,

- $\quad$ responding to changing work and commuter patterns with pop-up infrastructure that facilitates local neighbourhood working and invigorates local communities.
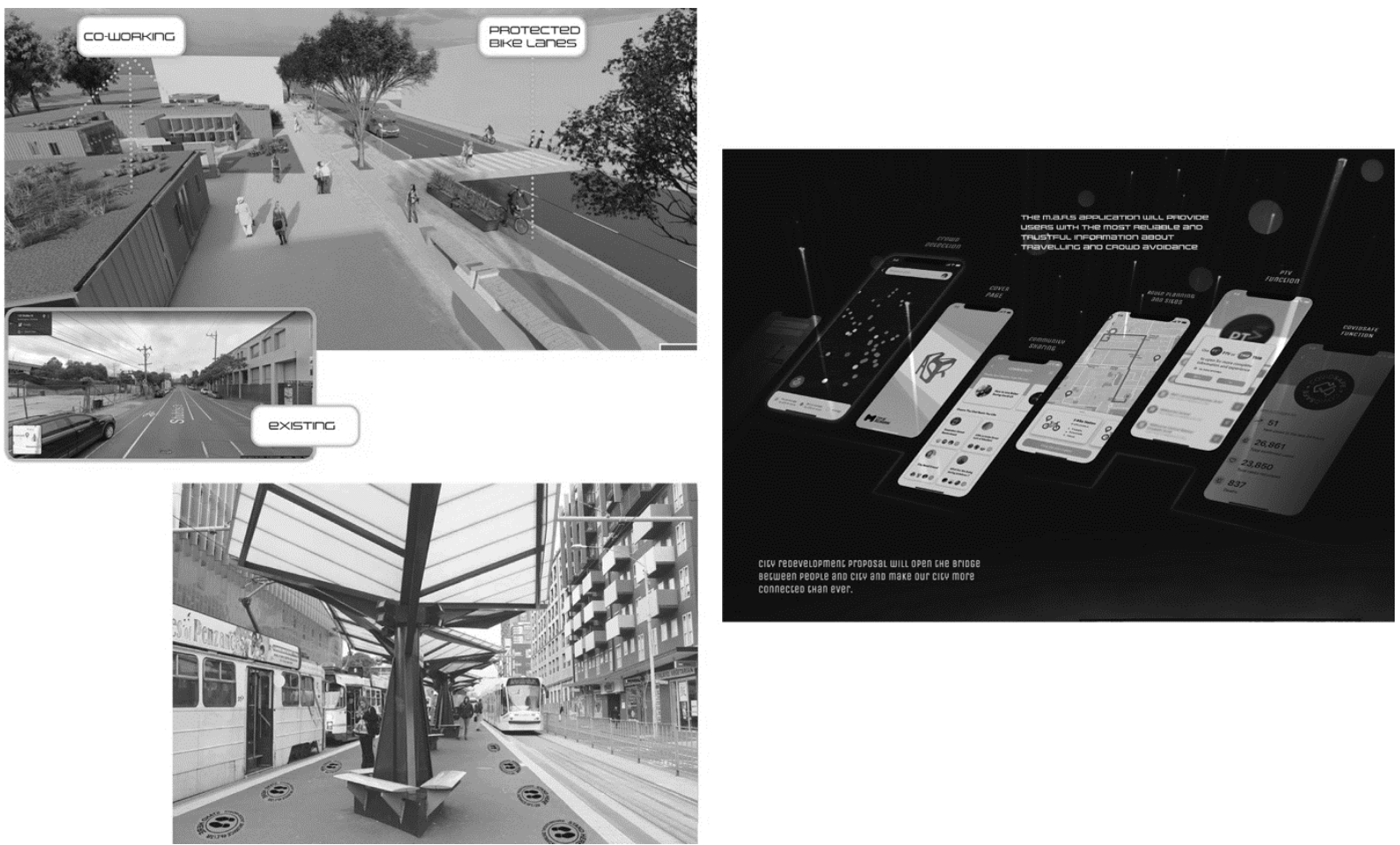

Figure 3. Safe Mobility with Covid-19 project design proposals

(clockwise from top left: Co-Op community co-working hub and neighbourhood rejuvenation, M.A.R.S safe urban navigation system, public transport social distancing system 


\section{FUTURE DIRECTIONS}

The Safeness by Design theme has proved valuable in design education. Through studio-based projects, students have had the opportunity to engage with real world issues and turn a reflective lens on urban design and examine human behaviours from a different perspective. The projects conducted so far have been successful and well received by students and external partners and have prompted new discourses. Designing with a safeness agenda has unlimited scope of possibilities. Even whilst avoiding the safety compliance or risk management fields of practice, all workplace and lifestyle environments have the potential for design to contribute more significantly to either actual or perceived safety. For future projects, the Safeness by Design team are moving their attention to the safeness of workers in the gig economy (notably food delivery workers) and also looking to reduce drowning and the frequency of life saving rescues of those who are unfamiliar with the dangerous surf conditions of Australia.

\section{CONCLUSIONS}

"Could the creation of well-being and not goods and services, be a new purpose for design?" With this question, Faud-Luke [5] proposes a model of 'design activism' in which designers are using "the power of design for the greater good." It is apparent that paradigm shifts have occurred in Industrial Design with practice moving towards discourses of practice that are societal and environmentally focused, resulting in new opportunities for both a broader contribution, and new currencies of value adding [6]. The World Design Organisation [7] states that Industrial Design is now "a strategic problem-solving process that...leads to a better quality of life through innovative products, systems, services, and experiences." Manzini [8] similarly sees the role of designers as facilitators who prompt new social conversations, and act as design activists proactively launching socially meaning design initiatives.

The Safeness by Design initiative responds to these calls with a new focus for design practice. Precedented by sustainable design, inclusive design, socially responsible design etc., which although specialisations, are now considered to be embedded within design practice, design for safeness is an important new consideration with potential for social impact. Not as a workplace risk management or compliance tool, but as a new approach to understanding, predicting, and responding to human behaviours to inform a more thorough design process.

Is Safeness a new paradigm of design intent?

\section{ACKNOWLEDGEMENTS}

The authors acknowledge project partners, the City of Melbourne and the Ajuntament de Barcelona and the role of RMIT Europe in facilitating the Catalan collaboration, and the associated study tour. We would like to give thanks and recognition to the students who responded to the project briefs with enthusiasm and dedication, achieving insightful and impactful outcomes.

\section{REFERENCES}

[1] Lloyd's Register Foundation. Foresight Review on Design for Safety: Protecting lives from the start, 2018, Lloyd's Register Foundation Report Series No. 2018.2

[2] Houssin, R., Coulibaly, A. An approach to solve contradiction problems for the safety integration in innovative design process. 2011. Computers in Industry, 62(4) pp398-406.

[3] Glendon, A.I., Stanton, N.A. Perspectives on safety culture, 2000, Safety Science, 34(1-3), pp.193214.

[4] Sadeghi, L., Dantan, J-Y., Mathieu, L., Siadat, A., Aghelinejad, A.M.M. A design approach for safety based on Product-Service Systems and Function-Behavior-Structure, 2017, CIRP Journal of Manufacturing Science and Technology, 19, pp 44-56.

[5] Fuad-Luke, A., Design activism: Beautiful strangeness for a sustainable world. 2009: Earthscan.

[6] de Vere, I., Fennessy, L. (2019) Redefining Industrial Design: Responding to Emerging Practice, in Towards a New Innovation Landscape, the 21st International Conference on Engineering and Product Design Education (E\&PDE2019), University of Strathclyde, Glasgow.

[7] World Design Organisation. Industrial Design Definition History. 2019 [cited 201915 February 2019]; Available from: https://wdo.org/about/definition/industrial-design-definition-history/.

[8] Manzini, E., Making Things Happen: Social Innovation and Design. Design Issues, 2014. 30(1). 